\title{
Application of Visual Management in Workshop
}

\author{
Mingyue Hao* \\ School of Economics and Management \\ Beijing Jiaotong University \\ Beijing, China \\ hmyzzia080507311@163.com \\ *Corresponding author
}

\author{
Zehua Wei \\ Management and Economic Department \\ Tianjin University \\ Tianjin, China \\ weizehua@yeah.net
}

\author{
Yuan Tian \\ School of Economics and Management \\ Beijing Jiaotong University \\ Beijing, China \\ tianmengfei@126.com
}

\begin{abstract}
In recent years, more and more enterprises begin to implement lean manufacturing and management. Enterprises try to reduce waste, costs and improve their efficiency in the production site. Production site is the main focus of lean manufacturing. Visual management as a method of site management creates a "transparent" workplace and plays an immediate effect on the improvement of production.
\end{abstract}

\section{Keywords-workshop; visual management}

\section{CONNOTATION OF Visual MANAGEMENT}

Visual management is a visible way of management with the use of visual perception information to guide, standardize and organize the production, aiming to ensure safety of production and improve labor productivity, make the management system visual, intuitive, simple, promote overall self-consciousness and initiative of observing discipline , get everybody autonomous management and self-control.

As a management method, visual management was initially applied to the scene of the homework with the style of color, label, sign and so on, so as to improve labor productivity, to distinguish the operation personnel, clear use state of instrument, process equipment and the dangerous state of production place. And it is also a kind of scientific method through vision management.

The contents of visual management include rules and regulations and work standard openness; the production task and completion figurization; combined with set management to realize visual display of information standardization; production control means image intuitive and use convenient; items packed and shipped quantity standardization; the field staff uniform and a system of hanging version; the standardization of color management.

\section{The MAIN IMPLEMENT MEANS OF VISUAL MANAGEMENT}

Visual management is a way of management by using all kinds of visual information and the scene of the sensory information with visual image and suitable color, to organize production activities, in order to improve the labor productivity.

Set management, board management, color management and identify management as the main means of visual management has obtained good effect in site management of the enterprise.

\section{A. Set Management}

"Set" of Set management is not literally "set goods at the fixed place", it refers to place items scientifically in a purposeful, planned, methodical way, according to restriction condition, such as the purpose of the production activities, considering the efficiency of the production activities, the quality etc. and the special requirements of the item itself (such as time, quality, quantity, process, etc.), then divide the appropriate places, determine the state of items in place to ensure production activities effectively.

Set management set the production site as the main object, research and analyze the conditions of people, objects, places and the relationship between them, and promote combination of people, machines, materials, systems and environment by arranging, consolidating, improving the conditions of production site. It mainly includes that dividing the production site into several areas in accordance with the process and placing each region; implementation of set of items and documentation within the range of the various functional departments; implementation of set of turnover equipment, products, materials on the production site (including warehouses, transport room), the weak links that affects the quality and safety (including flammable, perishable, toxic substances, etc.) be set management.

\section{B. Board Management}

Board management is a management method, which revealed project (information) that is hoped to manage through all kinds of management board, making the management status known to all. The particularity of environmental factors in production site determines the characteristics of the board management, which are striking, be clear at a glance, easy to 
use and management. As the board pointed out the spare parts, production, production time and methods, the name of the shipping and quantity and other relevant content, production site personnel can judge and handle problems independently. The kinds of board include standardized operation state of board, management board, state board and temporary board and so on. All kinds of board are convenient to control production capacity of process, inventories, and personnel arrangement and other circumstances, improving the effect of the operation and management.

\section{Color Management}

Color management is a management method using the psychological reaction and habits of people to color and resolution and association ability. It covers the management activities within the enterprise and management of physical with a layer of color coat. Staffs combine different color with the traffic lights naturally and consciously, so that every one of us has the same understanding and interpretation of a problem. When problems arise, they reach a consensus through language communication and determine the direction of the future. For example the production progress status is distinguished with color. Green stands for progress normally; Blue indicates production behind; Yellow indicates production to be expected, and red stands for mechanical failure. In addition, the application of color management can also include important parts of management, pipeline management, personnel management, content management, mold management, file management and raw material management.

\section{Identify Management}

Identify management manage personnel, materials, equipment and so on, mainly through the use of corresponding marks. In terms of personnel recognition, the larger the company scale is, the more need for personnel identify in order to work smoothly. Job qualified and skilled employee identification and other types are usually distinguished by coat color, epaulettes, tags and eye-catching signs. In terms of material identification, one of the most prone to errors project of the scene is material identification, which include name, number, quantity, origin, state recognition, good product and bad product identification, the identification of safekeeping conditions. Equipment identification mainly includes the equipment name, number, precision calibration management, operating personnel, maintenance personnel, operation condition, equipment, safety emergency escape, life device, operation flow signal in visual management. In addition, identify management includes job recognition, environment identification, non-conforming product identification, secure identification of visual management.

\section{VISUAL MANAGEMENT IMPLEMENTATION APPROACH OF PRODUCTION WORKSHOP}

\section{A. Workshop Management Visualizing}

\section{1) Policy objectives Visualizing}

Formulation of policy objectives, directly reflects the direction of the work of the workshop, strict small extent and general requirements. To enhance the full safety philosophy and create a good safety culture, the principle of management and objectives can be made into a huge banner hanging prominently in the workshop , reminding staff the contents of policy objectives in mind, thus will have effect on the promotion of "everyone knows goals and everyone works for them " management pattern's formation.

Leaders at all levels should promote the implementation of safety management, strengthen the model force, and improve the influence. Security work the leaders at all levels need to do can be made into a "personal safety action publicity panels". They should also regularly put on the implementation of major safety work and accept supervision by the masses. Through the adoption of these measures, leaders at all levels are able to take the initiative to carry out the required safety and environmental protection and further improve the work of consciousness.

\section{2) Documentation Visualizing}

In practical work, sometimes management staff's negligence causes the confusion of file data management, resulting in the inconvenient to take when they are used and inspected, and even the phenomenon of missing and lost. In this regard, the workshop documentation's uniform grading, sorting archiving and set- storage are needed. Paste the number on the box label and documentation index directory in each file to enable managers to collect and find files faster and more convenient to solve the problem of confusion documentation management, thus improving work efficiency.

\section{3) Territorial management Visualizing}

Territorial management is a new management method to establish responsibility system, and its essence is that every job site employee be responsible for the work of their own jurisdictions. This method can fully mobilize the enthusiasm of staff involved in management. You can hang territorial management map with different colors at the job site, and assign work areas, equipment, facilities etc down to each individual, then each staff will have the territorial scope of management and clear territorial management responsibilities. Visual work of territorial management enables staff positions more intuitive, territorial management be grasped in-depth and carried out easily. Then the work of management level is more clear and the implementation of management responsibilities are more effective.

\section{4) Emergency management Visualizing}

Emergency plan was developed to take quick rescue operation when faced with an emergency to reduce the losses to a minimum. How to make plans better implement and how to find the plan content the first time in the face of unexpected events, and promptly launch the emergency procedures of this problem can be solved by hanging " emergency management chart " which contains emergency management agencies, responsibilities and disposal procedures in the levels of the organization's emergency response office. In case of emergencies, you can ensure that there is principles can abide by.

\section{B. Personnel Assigned Visualizing}

Man as the carrier of construction and production, the important and indispensable component of whole social 
activities is especially important in the obvious visual management.

Personnel visual management should first start from scratch. According to the categories of persons in the workshop, different types of people wear helmets in different colors. Each individual's identity and responsibility to fulfill can be distinguished by the helmet's color.

Personnel assignment is fundamentally promote the cooperation of people and things and interpersonal coordination and workshop personnel assigned visualizing can reduce the time and energy waste to improve labor productivity.

\section{Equipment Visualizing}

Making the appropriate device status card, management card for the device belonging to the workshop that scrapped, owned, in the accounts. Device Management card indicates the device name, number, persons that be responsible for running and other relevant information. Device Status card display standby, disable, awaiting repair and the repair status of the device.

Strengthen basic management device, all of the device status (disabled, to be repaired, scrap), persons responsible for the device and various device safety inspection certificate such as the implementation of listing system to enable employees fully understand the device performance and the state. For forklifts, cranes and other mobile equipment should designate special parking area and clearly marked on them. By taking these measures, in regulating device management while improving site safety standardization and job security, reducing employees 'misuse' incidence while also helping employees develop good work habits.

Distribution box, drills and other electric equipment should be visibly marked "Electrical Hazard"、 "do not close" signs, giving obvious warning to operators other than electricians. Explosive storage containers, the use of fire-fighting device and the management of key fire zone must carry out a visual work too. Fire equipment and facilities should have periodic inspection by the person responsible for and implementation of listing system.

Production tools should be classified according to categories, located according to the predetermined position. According to the main tools in the toolkit, we should identified them outside of the toolkit. For example, if the toolkit has the tools such as wrench, screwdriver, hammer in it, it should have the above tools graphics and characters outside to explain. This kind of visual management makes workers get the tools accurately at the first time when looking for the tool before all the toolkit, thus avoiding the looking for links and improving the efficiency of the work.

\section{Workshop Layout Visualizing}

Workshop layout visualizing is using visual identity in the operating range and space to achieve the purpose of safety management. Orderly workshop layout can improve work efficiency, make people feel better, and achieve the result with half the effort.

\section{1) Dangerous equipment processing Visualizing}

In the process of dangerous equipment such as plate bending rolls and heating furnace, we should use Safety guardrail besides the marked warnings line. Safety guardrail has the appearance of horseshoe with two feet lotus connections. The color is given priority to with red and white or yellow and black. In normal times, guardrail is in a state of mutual connection to prevent people enter the danger zone. When the production needs, guardrail can temporarily open for the production. Such guardrail shape is simple and can be used conveniently and has a good warning role.

\section{2) Production areas Visualizing}

In the production areas, the workshop could be divided into a number of territorial management with own apanage management brand according to the work content and the layout of equipment. We can use the floor plan to mark each area clearly.

\section{3) Channel port of the workshop Visualizing}

In the workshop visual range, we should indicate security prohibited mark "no switching" and "no fireworks", "Pay attention to safety", security warning signs "be careful of hole" and "emergency exit", and safety tips "Must wear safety helmet" "must wear protective glasses". We should also indicate the type of work responsibility and bans, fire prevention, flood control, emergency plan, workshop safety escape diagram and workshop layout. In particular, we must make sure that each equipment has safety operation procedures. Visual management makes the safe production regulations easy to be abided and the equipment operation more easy and convenient.

\section{4) Office area Visualizing}

In office area of the visual range, we can indicate the handle with the logo "pull" and "push", the filing cabinets with the user's position and the main content of documents such as "contract management shoppe" "material purchasing" etc.

Through a series of security identity every link is in the "visual" and every detail has a "visible" reference, thus ensuring better safety in production.

\section{E. Operation Visualizing}

The concrete operation of staff should also be visual. Againsting illegal work has always been an important part of safety management work. Vast majority of industrial production safety accidents are caused by illegal. Every industrial production enterprises, therefore, will formulate a series of rules and embargo for the operation of the staff to make their behavior norm and standard. But the implementing of these regulations and restrictions does not reach the designated position in the actual work because of the imbalance master and understand or the uneven level of staff quality. Therefore, we can make standard process operation figure hanging in the appropriate location for employees to reference to make the activities more specific and standard. 


\section{CONCLUSION}

Through improving person, equipment, material, method of operation, production environment and other factors of production site, visual management can realize the rational allocation of production factors, the quick and fast transmission of information, the image intuitive show of potential problems and waste phenomenon of the scene. Visual management can make the job site safe and orderly, boost the morale of the employees, promote the establishment of enterprise culture and form, thus eliminating waste and reducing production cost, improving production efficiency and product quality, and enhancing the competitiveness of the enterprise itself.

\section{REFERENCES}

[1] Jianyou Hong, The application of visual management in the field of improving [J]. China quality report, 2012 (1) : 24-27.

[2] Sijin Tian and Jing Li, The innovation explore of Production safety work - "visual management" in the application of safety system [J]. Journal of safety, 2006, 12 (4) : 31-32.

[3] Fu Liu, Introduction about visual management to improve the logistics enterprises' production site [J]. Journal of motor practical technology, 2010, 23 (5) : 43-44.

[4] Jing $\mathrm{Li}$, Application of visual management in the field of improvement[D]. Dalian: Dalian university master's degree thesis, 2009.

[5] Moudu Lv, Business visual management [J]. Journal of Chinese computer users, 2008, 28 (13) :276-279. 